\title{
Cooperative Bargain for the Autonomous Separation of Traffic Flows in Smart Reversible Lanes
}

\author{
Guangbing Xiao $\mathbb{D}^{1},{ }^{1}$ Haibo Zhang, ${ }^{2}$ Ning Sun $\mathbb{D}^{1},{ }^{1}$ Yong Chen $\left(\mathbb{D},{ }^{3}\right.$ Jiamin Shi, \\ and Yong Zhang $\mathbb{D}^{1}$ \\ ${ }^{1}$ Department of Automobile and Traffic Engineering, Nanjing Forestry University, Nanjing 210044, China \\ ${ }^{2}$ Department of Computer Science, University of Otago, Dunedin 9046, New Zealand \\ ${ }^{3}$ Department of Mechanical and Electronic Engineering, Nanjing Forestry University, Nanjing 210044, China
}

Correspondence should be addressed to Yong Chen; chenyongjsnj@163.com

Received 24 April 2019; Accepted 4 October 2019; Published 30 October 2019

Guest Editor: Marin Lujak

Copyright ( $) 2019$ Guangbing Xiao et al. This is an open access article distributed under the Creative Commons Attribution License, which permits unrestricted use, distribution, and reproduction in any medium, provided the original work is properly cited.

\begin{abstract}
Spacer bars in the smart reversible lanes make periodically broadcast of messages to share their local observed traffic information with each other. This aims to help other spacer bars acquire the global traffic information and make consistent movement when separating the flows. However, radio interference and vehicles in the traffic may degrade the qualities of wireless communication links and cause frequent message losses in the broadcast. Existing solutions tend to use data forwarding to enhance the message dissemination, which may cause imbalanced load in the spacer bars. For instance, the nodes close to the sink have to forward more messages, whereas the ones far away from the sink have fewer messages for forwarding. The unbalanced distribution of network load has a high risk of blocking the wireless communication links and yield inconsistent movement in the reversible lanes. In this paper, we propose a Cooperative Bargain $(\mathrm{CoB})$ scheme where each spacer bar carries some received messages to help other spacer bars recover their lost messages. Since the spacer bars can only acquire the local information, we formulate a cooperative bargain game to negotiate how to allocate the task of message recovery with a balanced network load until a consensus is achieved. CoB is evaluated with the real-world Wi-Fi communication traces in Isti/rural. Simulation results show that CoB can recover an average of $98.6 \%$ messages within 100 milliseconds in a 50-node network. CoB does not require the global network information but it can still achieve a comparable performance to other broadcast schemes.
\end{abstract}

\section{Introduction}

The rapidly growing demand on congestion-free driving makes the smart traffic control a critical functionality in intelligent transportation systems (ITSs) [1,2]. To make full utilization of road resources, the smart reversible lanes are deployed on roads to reduce directional traffic congestion in rush hours. The smart reversible lanes monitor traffic environment and work as movable centre dividers to separate traffic flows from different directions, aiming to increase the traffic capacity in the peak direction by borrowing some unused lanes from off-peak direction. As shown in Figure 1, a smart reversible lane is composed of several spacer bars that are connected with longitudinal barriers. There are two categories of spacer bars in a smart reversible lane, i.e., active spacer bars and passive spacer bars. The active spacer bar is equipped with a radio transceiver, integrated sensors (e.g., video camera and ultrasonic unit), and a step motor. The radio transceiver is used for wireless communications, and the integrated sensors are used for monitoring the traffic environment. The step motor is installed on the base and powered by batteries to drive the spacer bar forward or backward. In contrast, the passive spacer bar has no radio transceiver, integrated sensors, or step motor. It is mechanically connected with the adjacent spacer bars and moves passively when dragged by others.

Considering the hardware investment and maintenance cost, the number of active spacer bars are limited and they 
are sparely distributed in the smart reversible lane. These active spacer bars form a backbone and drive the smart reversible lane forward or backward when separating the traffic flows. Each active spacer bar monitors the local traffic conditions, e.g., the volume of traffic flow and average vehicle speed, and then decides to move forward, backward, or stand still. Since the active spacer bars are distributed in a decentralized environment, they can only observe the local traffic information. This may yield dispute in the moving decisions and cause inconsistent movement of spacer bars as well as mechanical damages to the reversible lanes [3]. To eliminate the dispute in the moving decisions, the active spacer bars broadcast some messages to exchange their local observed traffic information with others and negotiate how to move the reversible lane consistently. However, the empirical studies in [4] have shown that, even in rural areas, the radio jamming and multiple-path interference can decrease the packet delivery ratio (PDR) by 50\%. Put it in another way, not all the active spacer bars can receive the messages. Since each message contains the local observed traffic information, the frequent message losses will cause inconsistent movement of the spacer bars and mechanical damages to the reversible lanes.

A straightforward approach to address this issue is data forwarding. Take a line network for example. The node forwards its received messages to the next node until they are successfully delivered to the sink. However, it will cause unbalanced load in the network, e.g., the nodes that are close to the sink have more messages to forward, whereas the nodes that are far away from the sink have few messages to forward [5]. In [6], a flooding-based scheme is proposed where each node forwards all received messages to help other nodes recover their lost ones. However, the fast expense of message duplications will increase the communication overhead and chock the communication channels with a high risk of broadcast storms. Another frequently used method is cooperative broadcasting, where the task of message delivery is offloaded to each node regarding its local resources, e.g., the quality of communication links and the remaining energy in the battery. Based on this key idea, a number of cooperative broadcasting schemes are proposed with different metrics for the task offloading, e.g., the geolocation-based schemes [7], the energy-based schemes $[8,9]$, and the neighborhood-based schemes [10, 11]. Note that most of these schemes require the global information for central administration and task offloading, which is challenging in wireless networks due to the unstable qualities of communication links.

In this paper, we develop a Cooperative Bargain (CoB) scheme to improve the message delivery ratio (MDR) in the broadcast. $\mathrm{CoB}$ only uses the local information at the active spacer bars but can reduce most disputes in the moving decisions and avoid inconsistent movements in the smart reversible lanes. Each active spacer bar carries some received messages to help others recover their lost messages. Since the messages are carried in each active spacer bar's broadcast, no extra retransmission is needed, yielding high MDRs with small communication overhead and short time delay. Besides, a decentralized cooperative bargain game is formulated to offload the task of message recovery regarding each active spacer bar's local resource [12]. The active spacer bars keep on negotiating with each other on which messages to carry until a consensus is achieved, so that they can make consistent movement in the reversible lanes.

The rest of this paper is organized as below. Section 2 reviews some related works on the reliable broadcast in wireless networks. Section 3 describes the system model and formulates the procedure of cooperative broadcast as an integral optimization problem. In Section 4, a cooperative bargain game is formulated to offload the task of message recovery. Section 5 proves that the achieved consensus in the cooperative bargain game is an optimal solution based on the theory of Nash equilibrium. Section 6 presents the simulation results where $\mathrm{CoB}$ is compared with some other broadcast schemes, and Section 7 concludes the whole paper.

\section{Related Work}

There have been active research studies on the reliable broadcast in wireless networks, but few can be applied to the wireless communications in smart reversible lanes. The reasons are twofold: (1) the signal jams and multiple-path interference in the traffic may degrade the qualities of wireless communication links between the spacer bars, causing frequent message losses in the broadcast of messages $[13,14]$ and (2) the active spacer bar can only obtain incomplete network topology and local traffic information, which may yield inconsistent moving actions and mechanical damages to reversible lanes [15]. Existing solutions can be divided into two categories, i.e., cooperative broadcast and noncooperative broadcast, depending on whether the nodes cooperate with others during the message recovery.

Flooding is a widely used noncooperative broadcast solution, where each node forwards all received messages through all outgoing channels to improve the broadcast reliability [16]. To reduce the communication overhead resulting from excessive duplications in the broadcast, some variants of flooding are proposed. As shown in [17], Dash et al. proposed a hop-controlled flooding to restrict the number of hops when forwarding the received messages. It describes a novel hop-count update procedure using a history database called Info-Base. Current hop-count update procedure reduces the hop count at each routing node. However, the inherent redundant duplication in the flooding-based schemes remains unchanged and the nodes still suffer from the high risk of broadcast storms. In [18], Byeon et al. proposed an opportunistic flooding-based scheme where the duplication of messages is strictly limited. Depending on the contribution level for the entire network, the proposed technique enhances transmission efficiency through priority adjustment and the removal of needless relay nodes. However, it is prone to blind spots or redundant overlaps if the parameter $p$ is not well designated, as it highly depends on the broadcast environment and application scenarios. 


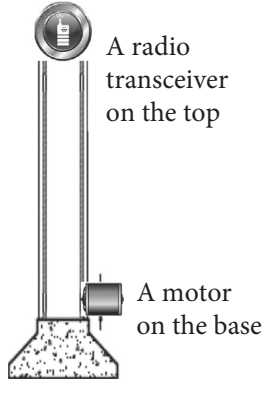

(a)

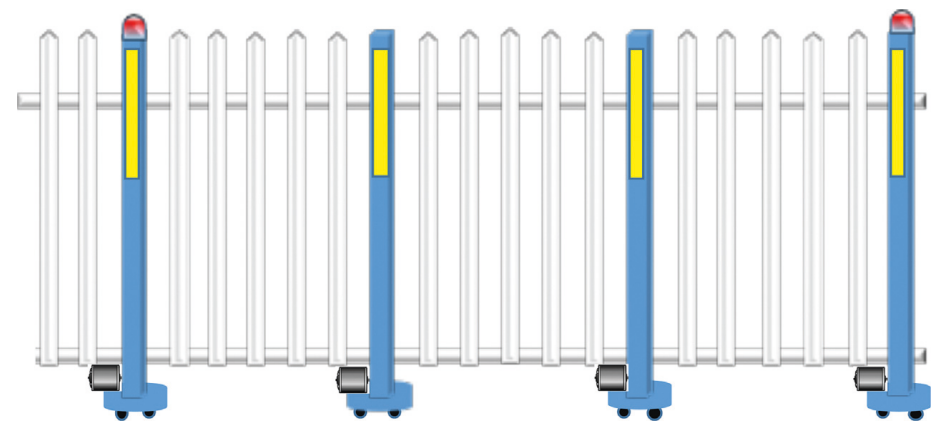

(b)

Figure 1: (a) The mechanical structure of an active spacer bar. (b) A smart reversible lane where the spacer bars are connected with longitudinal barriers.

The greedy-based scheme is a typical noncooperative solution for reliable dissemination of messages, where each node tries to recover the lost messages individually with little or seldom cooperation with others [19]. Xiao et al. [20] proposed a greedy-based piggybacking scheme to recover Cooperative Awareness Messages (CAMs) in the vehicular networks. Each vehicle broadcasts a request list to inform others which CAMs it has lost, and the CAM with maximum request-times will be selected for piggybacking first. However, the vehicles act too much greedy and little cooperation can be observed in the recovery of lost messages, which may cause redundant piggybacking and a low MDR with long time delay and large communication overhead. In [21], Xu et al. formed a noncooperative game for distributed wireless sensor networks to control the topology for energy saving and network load balancing. A price function is developed to calculate the reward for forwarding messages and the penalty for declining messages in the broadcast. Since all the nodes tend to maximize the reward, both the MDR and the network lifetime can be enhanced when a Nash equilibrium is achieved. However, Nash equilibrium is not sufficient to guarantee a global optimal solution. A simple example is the prisoner's dilemma in the game theory.

Recently, much attention has been paid to the cooperative broadcast, where the message recovery is offloaded to the nodes in a cooperative manner [22, 23]. For instance, a cooperative beacon broadcast scheme is proposed in [24] to provide the vehicles with more traffic information when driving on roads. Each vehicle selects $w$ neighbors and piggybacks their awareness messages in the periodical beacon broadcast. It aims to improve the driving safety via the exchange of traffic information as it can provide sufficient traffic awareness when driving on roads. The drawback is that each vehicle has to dynamically maintain the geographic information of all its neighboring vehicles, which will consume a lot of network resources and introduce large communication overhead in the networks. A cooperative volunteer-based broadcast is studied in [25], where each vehicle exchanges its routing table with others to explore hidden neighbors in the non-line of sight (NLOS) area. The vehicles that have reliable links to the hidden neighbors will be selected as forwarders to retransmit the messages. Similarly, this scheme assumes that all nodes can obtain the global network information when offloading the task of message recovery, which is quite challenging for the active spacer bars in smart reversible lanes $[26,27]$.

This paper provides a novel cooperative broadcast scheme called $\mathrm{CoB}$ to improve MDR in the broadcast of messages, aiming to eliminate the bias and making consistent movements in reversible lanes. Besides, a cooperative bargain game is formulated to negotiate the recovery strategies and moving decisions in all nodes until a consensus is achieved. Therefore, the task of message recovery can be offloaded to the nodes regarding their local resources, which is proved to be a global optimal solution to the cooperative recovery problem. Since the messages can be delivered reliably and efficiently, the spacer bars will acquire the same traffic information and thus move consistently to separate the traffic flows.

\section{System Model and Problem Statement}

Consider a smart reversible lane with $|N|$ active spacer bars. Each active spacer bar is equipped with a radio transceiver for transmitting and receiving messages, and the radio transceivers have the same transmission range of $r$ meters. A stepping motor is installed on the base of each active spacer bar to move it forward or backward for separating the traffic flows. We model the smart reversible lane as a line network $\mathbb{G}=(N, L)$. The vertices in $N=\left\{n_{i}|i \leq| N \mid, i \in \mathbb{Z}^{+}\right\}$represent the active spacer bars, which is referred to as node(s) in the rest of this paper. The edges in $L=\left\{l_{i j}|i, j \leq| N \mid, i\right.$, $\left.j \in \mathbb{Z}^{+}, i \neq j\right\}$ denote the wireless communication links between the active spacer bars.

Time is synchronized within a consensus-based synchronization method. Each node uses the carrier sense multiple access/collision detection (CSMA/CD) scheme to access the channels for broadcasting, which can achieve a high utilization of channel resources which do not require the central administration to schedule the nodes for broadcasting [28]. Once the local traffic conditions have changed (e.g., the vehicle density has changed by 0.1 vehicle/ $\mathrm{m}$ and the shockware speed has changed by 2 vehicle/s), the 
node will broadcast a message to inform other nodes of the up-to-date local traffic information [29]. Thereby, the nodes will acquire more traffic information to make consistent movement in the reversible lanes when separating the traffic flows. The messages received by node $n_{i}$ are cached in its receiving buffer $F_{i}$. Each message is associated with a timeto-live (TTL) of $\delta \mathrm{ms}$ and will be discarded when the TTL expires.

However, not all the nodes can receive the messages due to the fragile communication links, which may cause inconsistent movements in the reversible lanes when separating the traffic flows. To address this issue, each node carries some received messages when it is scheduled for broadcasting and helps other nodes recover their lost messages. Let $s_{i}^{t}$ be node $n_{i}$ 's task in the message recovery, and the combination of each node's task in the message recovery, $\delta_{i}^{t}=\left\{s_{1}^{t}, s_{2}^{t}, \ldots, s_{|N|}^{t}\right\}$ is defined as the joint recovery strategy in the broadcast. Suppose $M_{j}$ is the set of messages generated by node $n_{j}$ in the broadcast, and $\left|N_{i}\left(M_{j}, \mathcal{S}^{t}\right)\right|$ is the number of messages in $M_{j}$ that are received by node $n_{i}$ under the joint recovery strategy $\delta^{t}$. Then, node $n_{i}$ 's message reception ratio (MRR) regarding node $n_{j}$ 's messages, denoted by $r_{i j}\left(\mathcal{S}^{t}\right)$ $=\left|N_{i}\left(M_{j}, \delta^{t}\right)\right| /\left|M_{j}\right|$, is defined as the ratio between the number of messages received by node $n_{i}$ and the total number of messages in $M_{j}$. Thereby, we can convert the process of cooperative broadcasting in the reversible lane into an integral optimization problem as shown in the following equation:

$$
\max \sum_{n_{i} \in N} \frac{\sum_{n_{j} \in N} r_{i j}\left(\mathcal{S}^{t}\right)}{|N|^{2}} .
$$

Equation (1) indicates that the joint recovery strategy $\mathcal{S}^{t}$ should be capable of maximizing the average MRR for all the nodes in the broadcast. It aims to find an optimal joint recovery strategy $\mathbb{S}^{t *}$ that can maximize the MRR in the broadcast, so that they can acquire the same messages and make consistent movement when separating the traffic flows.

\section{Cooperative Bargaining}

In this section, we formulate a cooperative bargain game to allocate the task of cooperative recovery regarding each node's local resources, and then propose a Cooperative Bargain scheme to maximize the objection function in 1 .

4.1. Game Formulation. To achieve a consensus in the recovery of lost messages, each node $n_{i}$ generates a bargain proposal $B_{i}^{t}$ and exchanges it with other nodes for negotiation. The bargain proposal contains a recommending strategy $\mathscr{R}_{i}^{t}$ and a bidding strategy $\mathscr{P}_{i}^{t}$. The recommending strategy $\mathscr{R}_{i}^{t}=\left\{r_{i 1}^{t}, r_{i 2}^{t}, \ldots, r_{i|N|}^{t}\right\}$ is a set of recommendations generated by node $n_{i}$, and it indicates which message node $n_{i}$ expects other nodes to carry. The bidding strategy $\mathscr{P}_{i}^{t}=\left\{p_{i 1}^{t}, p_{i 2}^{t}, \ldots, p_{i|N|}^{t}\right\}$ is a set of bidding prices offered by node $n_{i}$ corresponding to each recommendation in $\mathscr{R}_{i}^{t}$. The higher bidding price the node $n_{i}$ offers, the higher priority the recommended message will be piggybacked first.

After generating a bargain proposal, each node will receive a reward, depending on how much its bargain proposal deviates from other nodes' bargain proposals. The bargain proposal with less deviation will yield more rewards and vice versa. To maximize the rewards, the nodes will negotiate with each other to update their bargain proposal until a consensus is achieved. The deviations of bargain proposals are referred to as bargain bias in the rest of this paper, and the definition is presented as below:

Definition 1. Consider two bargain proposals generated by node $n_{i}$ and $n_{j}$ at time $t$, respectively, i.e., $B_{i}^{t}=\left\{\mathscr{R}_{i}^{t}, \mathscr{P}_{i}^{t}\right\}$ and $B_{j}^{t}=\left\{\mathscr{R}_{j}^{t}, \mathscr{P}^{t}\right\}$. The bargain bias of $B_{i}^{t}$ against $B_{j}^{t}$, denoted by $\theta_{i j}^{t}$ as shown in equation (3), indicates how much node $n_{i}$ 's bargain proposal deviates from node $n_{j}$ 's bargain proposal:

$$
\theta_{i j}^{t}=\left(\mathscr{P}_{j}^{t}-\mathscr{P}_{i}^{t}\right) \cdot(1)^{T}+\left(\mathscr{R}_{j}^{t}-\mathscr{R}_{i}^{t}\right) \cdot \operatorname{diag}\left(\mathscr{P}_{i}^{t}\right) \cdot\left(\mathscr{R}_{j}^{t}-\mathscr{R}_{i}^{t}\right)^{T} .
$$

According to Definition 1 , if nodes $n_{i}$ and $n_{j}$ have generated the same bargain proposal, e.g., they have made the same recommending strategy and offered the same bidding prices, the bargain bias is calculated as 0 ; otherwise, the bargain bias is nonzero. To make consistent movement in the reversible lanes, the nodes should eliminate the bargain bias and enforce all the bargain proposals converge to a consensus. The challenge is that the nodes can only acquire the local information, and it is difficult to compare the bargain proposal with all the other nodes in the decentralized environment. To address this issue, we connect all the nodes into a virtual ring and divide them into several overlapped groups. Each group is composed of three nodes, e.g., a heading node, a central node, and an ending node, and the group overlaps with a preceding group and a succeeding group, respectively. Put it in another way, the heading node in the current group is the ending node of its preceding group, whereas the ending node is the heading node of its succeeding group. For example, consider a set of $|N|$ nodes in Figure 2. If $|N|$ is an odd number, the nodes can be divided as, $\left\{\left(n_{1}, n_{3}, n_{5}\right),\left(n_{3}, n_{5}, n_{7}\right), \ldots,\left(n_{|N|-4}\right.\right.$, $\left.n_{|N|-2}, n_{|N|}\right),\left(n_{|N|-2}, \quad n_{|N|}, \quad n_{|N|-1}\right), \quad\left(n_{|N|}, n_{|N|-1}, n_{|N|-3}\right), \ldots$, $\left.\left(n_{4}, n_{2}, n_{1}\right),\left(n_{2}, n_{1}, n_{3}\right)\right\}$. If the number of nodes $|N|$ is even, the nodes can be divided as $\left\{\left(n_{1}, n_{3}, n_{5}\right),\left(n_{3}, n_{5}, n_{7}\right)\right.$, $\ldots,\left(n_{m-5}, n_{m-3}, n_{m-1}\right),\left(n_{m-3}, n_{m-1}, n_{m}\right) \quad,\left(n_{m-1}, n_{m}, n_{m-2}\right)$, $\left.\ldots,\left(n_{4}, n_{2}, n_{1}\right),\left(n_{2}, n_{1}, n_{3}\right)\right\}$. Since each group overlaps with its preceding group and the succeeding group, the central node only has to compare its bargain proposal inside of the group because the preceding node and the successive node will carry on comparing the bargain proposal with other nodes in the overlapped groups. Thereby, the bargain proposals will be compared among all the nodes group by group along the virtual ring until a consensus is achieved. 


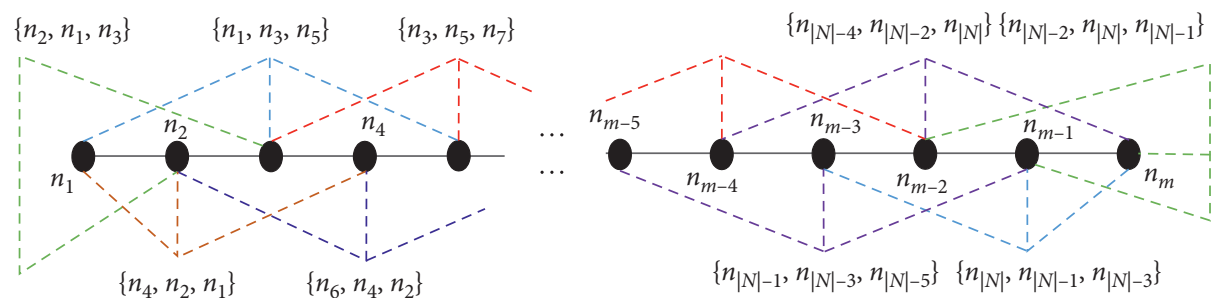

(a)
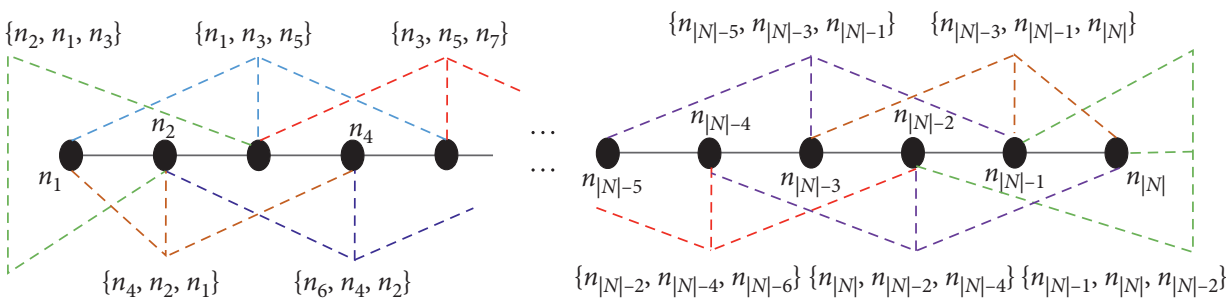

(b)

FIGURE 2: Dividing all nodes into groups for the comparison of bargain proposals. (a) The division of groups when the number of nodes $|N|$ is odd. (b) The division of groups when the number of nodes $|N|$ is even.

Definition 2. Consider a group with three nodes $\left(n_{i}, n_{j}\right.$, and $\left.n_{k}\right)$ in the cooperative bargain game. $B_{j}^{t}$ is the bargain proposal generated by the central node $n_{j}$. It will receive a reward $w\left(B_{i}^{t}\right)$, which is defined as the difference between the bargain bias $\theta_{i j}^{t}$ and $\theta_{j k}^{t}$ in the following equation:

$$
\begin{aligned}
w\left(B_{i}^{t}\right)= & \theta_{i j}^{t}-\theta_{j k}^{t}=\left(\mathscr{P}_{i}^{t}-\mathscr{P}_{j}^{t}\right) \cdot(1)^{T}+\left(\mathscr{R}_{i}^{t}-\mathscr{R}_{j}^{t}\right) \cdot \operatorname{diag}\left(\mathscr{P}_{i}^{t}\right) \\
& \cdot\left(\mathscr{R}_{i}^{t}-\mathscr{R}_{j}^{t}\right)^{T}-\left(\mathscr{P}_{j}^{t}-\mathscr{P}_{k}^{t}\right) \cdot(1)^{T}-\left(\mathscr{R}_{j}^{t}-\mathscr{R}_{k}^{t}\right) \\
& \cdot \operatorname{diag}\left(\mathscr{P}_{j}^{t}\right) \cdot\left(\mathscr{R}_{j}^{t}-\mathscr{R}_{k}^{t}\right)^{T} .
\end{aligned}
$$

As shown in equation (3), if the bargain bias between nodes $n_{i}$ and $n_{j}$ is less than the bargain bias between nodes $n_{j}$ and $n_{k}$, node $n_{i}$ will receive a positive reward $w\left(B_{i}^{t}\right)>0$ as a bonus for its bargain proposal; otherwise, node $n_{i}$ will receive a negative reward $w\left(B_{i}^{t}\right)<0$ as a penalty for its bargain proposal. Since each node aims to maximize the rewards, the bargain bias will be eliminated ultimately. However, eliminating the bargain bias does not mean the messages have been recovered successfully, e.g., the nodes may achieve a consensus that no one piggybacks any message. The nodes will not receive any penalty but they fail to recover the lost messages in the broadcast. To solve this problem, we combine the reward with MRR and form a utility function as shown in equation (4).

Definition 3. For each node $n_{i}$ in the cooperative bargain game, the utility function, denoted by $u\left(B_{i}^{t}=\left\{\mathscr{R}_{i}^{t}, \mathscr{P}_{i}^{t}\right\}\right)$, is defined as the sum of MRR following its recommending strategy $\mathscr{R}_{i}^{t}$ and the reward for its bargain proposal $w\left(B_{i}^{t}\right)$ :

$$
u\left(B_{i}^{t}\right)=\frac{\sum_{n_{j} \in N} r_{i j}\left(\mathscr{R}_{i}^{t}\right)}{|N|^{2}}+w\left(B_{i}^{t}\right)
$$

For each node in the cooperative bargain, it keeps on updating the bargain proposal to eliminate the bargain bias and maximize the utility function. According to the definition of Nash equilibrium in [30], all the bargain proposals will converge to a consensus, which is referred to as balance point in the Nash equilibrium. When the balance point is achieved, none of the nodes is willing to change its bargain proposal as the utility function has been maximized according to the node's local information.

4.2. Cooperative Bargain. In this subsection, a Cooperative Bargain ( $\mathrm{CoB})$ scheme is proposed to maximize the utility function in equation (4). The key ideas of $\mathrm{CoB}$ can be described as follows:

(1) For each message in the broadcast, node $n_{i}$ generates a set of recommendations to indicate which node it expects to carry the message. Considering all the messages in the broadcast, the combination of node $n_{i}$ 's recommendations to each other node, i.e., $\mathscr{R}_{i}^{t}=\left\{r_{i 1}^{t}, r_{i 2}^{t}, \ldots, r_{i|N|}^{t}\right\}$, is defined as its recommending strategy.

(2) For each recommendation in $\mathscr{R}_{i}^{t}$, node $n_{i}$ offers a bidding price. The higher bidding price it offers, the higher priority the recommended message is expected to be carried first. The set of bidding prices offered by node $n_{i}$, denoted by $\mathscr{P}_{i}^{t}=\left\{p_{i 1}^{t}, p_{i 2}^{t}, \ldots, p_{i|N|}^{t}\right\}$, is defined as its bidding strategy.

(3) The combination of piggybacking strategy and bidding strategy, i.e., $B_{i}^{t}=\left\{\mathscr{R}_{i}^{t}, \mathscr{P}_{i}^{t}\right\}$, is defined as node $n_{i}$ 's bargain proposal. Node $n_{i}$ compares the bargain proposal with the heading node and ending 
node in the group, and it calculates the reward $w\left(B_{i}^{t}\right)$ regarding the bargain bias between them.

(4) The node $n_{i}$ keeps on adjusting its bargain proposal to maximize the utility function until a consensus is achieved.

A key issue in $\mathrm{CoB}$ is how to generate and update the bargain proposals. If the nodes know the qualities of wireless communication links, they will recommend the one with a good link to piggyback the messages. Meanwhile, the bidding price can be offered regarding how many nodes have lost the message. The more nodes that have lost the message, the higher bidding price will be offered for recommending other nodes to carry it for recovery. To address this issue, we develop the following two principles for generating and updating the bargain proposals.

Principle 1. The nodes with fewer stable communication links should be recommended for recovering the messages first.

The nodes with more stable communication links tend to have more options when carrying the messages for recovery. If these nodes are recommended for recovery first, they may choose to carry some messages that can also be recovered by other nodes, but fail to carry the messages that can only be recovered by themselves.

Principle 2. If the bargain proposal is different from that of others, the node should

(1) Choose the mainstreamed one as the new bargain proposal if it can recover the message with fewer forwarders, shorter time delays, or higher success probability

(2) Remain the bargain proposal unchanged if the mainstreamed one fails to recover one or more messages

Since the nodes can only acquire the local information, they may generate different bargain proposals for the message recovery. To eliminate the bargain bias, each node should update its bargain proposal to achieve a consensus. Note that some nodes have already achieved a local consensus (e.g., the mainstreamed bargain proposal can be regarded as a local consensus), and they have a higher probability to make an optimal bargain proposal as they have acquired more information via exchanging the bargain proposals. Therefore, if a node has generated a bargain proposal that is different from the mainstream one, it should update its bargain proposal to coincide with the mainstreamed one. However, it might be possible that the node has detected a lost message which fails to be detected by the nodes with the mainstreamed bargain proposals. Therefore, the node should check whether the mainstreamed bargain proposal can recover all the messages. If the mainstreamed bargain proposal fails to recover one or more messages, the node should remain its bargain proposal unchanged on recovering these messages, and thus the other nodes will be informed that the mainstreamed bargain proposal failed to recover all the messages.

The pseudocode of $\mathrm{CoB}$ is given in Algorithm 1, where each node $n_{i}$ exchanges its bargain proposal with other nodes for comparison and updating until a consensus is achieved. Upon broadcasting a packet, each node $n_{i}$ will infer the lost messages and qualities of wireless communication links in the broadcast. Then, it will generate a bargain proposal based on the inferred information to maximize the utility function. Upon receiving a packet from another node $n_{j}$, node $n_{i}$ will check each piggybacked message in the packet and cache it in the receiving buffer $F_{i}$. The node $n_{j}$ 's bargain proposal in the received packet will be used to update node $n_{i}$ 's bargain proposal if it is different from the mainstreamed one.

\section{Theoretical Analysis}

In $\mathrm{CoB}$, each node generates bargain proposals based on its local information and then exchanges them with other nodes for negotiation and updating so that the bargain bias can be eliminated. In this section, we prove that the achieved consensus in CoB is Nash equilibrium (NE), and the bargain proposal at the NE is an optimal solution for the cooperative recovery of lost messages in the broadcast.

Theorem 1. If a consensus is achieved in the cooperative bargain game, the combination of each node's bargain proposal is a Nash equilibrium to the objection function in equation (1).

Proof. Suppose $\mathbb{R}^{t}=\left\{\mathscr{R}_{1}^{t}, \mathscr{R}_{2}^{t}, \ldots, \mathscr{R}_{|N|}^{t}\right\}$ is the set of recommending strategies generated by all the nodes at time $t$ and $\mathbb{P}^{t}=\left\{\mathscr{P}_{1}^{t}, \mathscr{P}_{2}^{t}, \ldots, \mathscr{P}_{|N|}^{t}\right\}$ is the set of bidding prices corresponding to each recommending strategy in $\mathbb{R}^{t}$. Denote the achieved consensus by $\mathbb{B P}^{t *}=\left\{B_{i}^{t *}=\right.$ $\left.\left(\mathscr{R}_{i}^{t *}, \mathscr{P}_{i}^{t *}\right) \mid n_{i} \in N\right\}$. Define $\mathbb{R}^{t *}=\left\{\mathscr{R}_{1}^{t *}, \mathscr{R}_{2}^{t *}, \ldots, \mathscr{R}_{|N|}^{t *}\right\}$ and $\mathbb{P}^{t *}=\left\{\mathscr{P}_{1}^{t *}, \mathscr{P}_{2}^{t *}, \ldots, \mathscr{P}_{|N|}^{t *}\right\}$ as the joint recommended strategies and bidding prices at the consensus, respectively. According to the definition of Nash equilibrium, the achieved consensus $\mathbb{B P}^{t *}$ is a balance point where no node tends to change its bargain proposal or bidding price unilaterally, where each node's utility function has been locally maximized. Considering the group division in Figure 2 and the definition of rewards in equation (3), the sum of rewards at each node is zero as shown in the following equation:

$$
\begin{aligned}
\sum_{n_{i} \in N} w\left(B_{i}^{t *}\right)= & \left(\theta_{13}^{t}-\theta_{35}^{t}\right)+\left(\theta_{35}^{t}-\theta_{57}^{t}\right)+\cdots+\left(\theta_{42}^{t}-\theta_{21}^{t}\right) \\
& +\left(\theta_{21}^{t}-\theta_{13}^{t}\right)=0 .
\end{aligned}
$$

By substituting equation (5) into the utility function in equation (4), we can convert the objection function into the following equation: 
//Upon broadcasting a packet:

Infer the qualities of wireless communication links and the lost messages in the broadcast;;

for each lost message in the broadcast do

Recommend the node with the fewest stable communication links to piggyback the message

if the recommendation is different from the mainstreamed one then

Rank all the received recommendations on piggybacking this message, and choose the top-ranked one as the new recommendation;

Broadcast a packet with the bargain proposal to inform other nodes of node $n_{i}$ 's recommendations

//Upon receiving a packet from node $n_{j}$ :

for each piggybacked message in the received packet do

if there is no such a message in the receiving buffer $F_{i}$ then

Cache it in the receiving buffer $F_{i}$.

else

Keep the one that has the latest time stamp.

Cache node $n_{j}$ 's bargain proposal for comparison;

Algorithm 1: CoB at each node $n_{i}$.

$$
\begin{aligned}
\sum_{n_{i} \in N} \frac{\sum_{n_{j} \in N^{r}} r_{i j}\left(\mathscr{R}_{i}^{t *}\right)}{|N|^{2}}= & \sum_{n_{i} \in N}\left(u\left(B_{i}^{t *}\right)-w\left(B_{i}^{t *}\right)\right)=\sum_{n_{i} \in N} u\left(B_{i}^{t *}\right) \\
& -\sum_{n_{i} \in N} w\left(B_{i}^{t *}\right)=\sum_{n_{i} \in N} u\left(B_{i}^{t *}\right) .
\end{aligned}
$$

Since each node's utility function achieves a Nash equilibrium at $\mathbb{B P}^{t *}=\left\{\left(\mathscr{R}_{i}^{t *}, \mathscr{P}_{i}^{t *}\right) \mid n_{i} \in N\right\}$, the object function in equation (1) will also achieve a Nash equilibrium at $\mathbb{B P}^{t *}=\left\{\left(\mathscr{R}_{i}^{t *}, \mathscr{P}_{i}^{t *}\right) \mid n_{i} \in N\right\}$ based on the equivalence relationship as shown in equation (6).

Theorem 1 proves that the achieved consensus is a Nash equilibrium in the cooperative bargain game. However, it does not mean that the achieved Nash equilibrium is the optimal bargain proposal. In other words, maximizing $u\left(B_{i}^{t *}\right)$ locally at each node is not equivalent to maximizing $\sum_{n_{i} \in N} u\left(B_{i}^{t *}\right)$ globally. The following Theorem 2 proves that the Nash equilibrium achieved in the cooperative bargain game is an optimal solution to the objective function in equation (1).

Theorem 2. The combination of each node's bargain proposal at the achieved consensus in the cooperative bargain game is an optimal solution to the objective function in equation (1).

Proof. Consider a bargain proposal $\mathbb{B P}^{t}=\left\{B_{i}^{t}=\left(\mathscr{R}_{i}^{t *}, \mathscr{P}_{i}^{t}\right) \mid\right.$ $\left.n_{i} \in N\right\}$ in the cooperative bargain game. It cannot yield a higher value in the object function than the one at the Nash equilibrium, i.e., $\mathbb{B} \mathbb{P}^{t *}=\left\{B_{i}^{t *}=\left(\mathscr{R}_{i}^{t *}, \mathscr{P}_{i}^{t *}\right) \mid n_{i} \in N\right\}$. Therefore, we can have the following inequality:

$$
\begin{aligned}
\sum_{n_{i} \in N} u\left(B_{i}^{t}\right) & \leq \sum_{n_{i} \in N} u\left(B_{i}^{t *}\right), \\
B_{i}^{t} & =\left\{\left(\mathscr{R}_{i}^{t *}, \mathscr{P}_{i}^{t}\right)\right\}, \\
B_{i}^{t *} & =\left\{\left(\mathscr{R}_{i}^{t *}, \mathscr{P}_{i}^{t *}\right)\right\}, \\
\mathbb{B P}^{t} & =\left\{B_{1}^{t *}, B_{2}^{t *}, \ldots, B_{i}^{t}, \ldots, B_{|N|-1}^{t *}, B_{|N|}^{t *}\right\}, \\
\mathbb{B P}^{t *} & =\left\{B_{1}^{t *}, B_{2}^{t *}, \ldots, B_{i}^{t *}, \ldots, B_{|N|-1}^{t *}, B_{|N|}^{t *}\right\} .
\end{aligned}
$$

Since the utility function $u\left(B_{i}^{t}\right)$ is strictly monotone decreasing of $w\left(B_{t}^{i}\right)$, we can have the following inequality:

$$
\forall n_{i} \in N, \quad w\left(B_{i}^{t}\right) \leq w\left(B_{i}^{t *}\right)
$$

Suppose node $n_{i}$ is the heading nodes in the group $\left(n_{i}, n_{j}\right.$, and $\left.n_{k}\right)$, and then we can rewrite Inequality (8) into Inequality (9) based on the definition of $w\left(B_{i}^{t}\right)$ :

$$
\left(\mathscr{P}_{i}^{t}-\mathscr{P}_{i}^{t *}\right)+\left(\mathscr{R}_{i}^{t}-\mathscr{R}_{j}^{t *}\right)^{T} \operatorname{diag}\left(\mathscr{P}_{i}^{t}-\mathscr{P}_{i}^{t *}\right)\left(\mathscr{R}_{i}^{t}-\mathscr{R}_{j}^{t *}\right) \leq 0 .
$$

Since Inequality (9) stands up for all bidding prices, we can have Inequality (10) by substituting $\mathscr{P}_{i}^{t}=2 \mathscr{P}_{i}^{t *}$ and $\mathscr{P}_{i}^{t}=0$ into Inequality (9), respectively:

$$
\mathscr{P}_{i}^{t *}+\left(\mathscr{R}_{i}^{t}-\mathscr{R}_{j}^{t *}\right)^{T} \operatorname{diag}\left(\mathscr{P}_{i}^{t *}\right)\left(\mathscr{R}_{i}^{t}-\mathscr{R}_{j}^{t *}\right)=0 .
$$

Substitute equation (10) into equation (3), and the reward of node $n_{i}$ at the Nash equilibrium is calculated as follows:

$$
w\left(B_{i}^{t *}\right)=\left(\mathscr{P}_{k}^{t *}-\mathscr{P}_{j}^{t *}\right)(1)^{T}
$$

Consider the definition of the Nash equilibrium again, and we can have another inequality (12) as below: 


$$
\begin{aligned}
\sum_{n_{i} \in N} u\left(B_{i}^{t^{\prime}}\right) & \leq \sum_{n_{i} \in N} u\left(B_{i}^{t *}\right), \\
B_{i}^{t^{\prime}} & =\left\{\left(\mathscr{R}_{i}^{t^{\prime}}, \mathscr{P}_{i}^{t^{\prime}}\right)\right\}, \\
B_{i}^{t *} & =\left\{\left(\mathscr{R}_{i}^{t *}, \mathscr{P}_{i}^{t *}\right)\right\}, \\
\mathbb{B P}^{t} & =\left\{B_{1}^{t *}, B_{2}^{t *}, \ldots, B_{i}^{t^{\prime}}, \ldots, B_{|N|-1}^{t *}, B_{|N|}^{t *}\right\}, \\
\mathbb{B P}^{t *} & =\left\{B_{1}^{t *}, B_{2}^{t *}, \ldots, B_{i}^{t *}, \ldots, B_{|N|-1}^{t *}, B_{|N|}^{t *}\right\} .
\end{aligned}
$$

By substituting Inequality (11) into Inequality (12), we can have the following inequality:

$$
\begin{gathered}
\sum_{n_{i} \in N} \frac{\sum_{n_{j} \in N} r_{i j}\left(\mathscr{R}_{i}^{t^{\prime}}\right)}{|N|^{2}}+\left[\left(\mathscr{P}_{i}^{t^{\prime}}-\mathscr{P}_{j}^{t *}\right)-\left(\mathscr{P}_{j}^{t *}-\mathscr{P}_{k}^{t *}\right)\right] \cdot(1)^{T} \\
\quad+\left(\mathscr{R}_{i}^{t^{\prime}}-\mathscr{R}_{j}^{t *}\right) \cdot \operatorname{diag}\left(\mathscr{P}_{i}^{t^{\prime}}\right) \cdot\left(\mathscr{R}_{i}^{t^{\prime}}-\mathscr{R}_{j}^{t *}\right)^{T} \\
\quad-\left(\mathscr{R}_{j}^{t *}-\mathscr{R}_{k}^{t *}\right) \cdot \operatorname{diag}\left(\mathscr{P}_{j}^{t *}\right) \cdot\left(\mathscr{R}_{j}^{t *}-\mathscr{R}_{k}^{t *}\right)^{T} \\
\leq \sum_{n_{i} \in N} \frac{\sum_{n_{j} \in N} r_{i j}\left(\mathscr{R}_{i}^{t *}\right)}{|N|^{2}}+\left(\mathscr{P}_{k}^{t *}-\mathscr{P}_{j}^{t *}\right)(1)^{T} .
\end{gathered}
$$

Since Inequality (13) holds for all values of $\mathscr{P}_{i}^{t^{\prime}}$, we can obtain Inequality 14 by setting $\mathscr{P}_{i}^{t^{\prime}}=0$ in Inequality (13):

$$
\sum_{n_{i} \in N} \frac{\sum_{n_{j} \in N} r_{i j}\left(\mathscr{R}_{i}^{t^{\prime}}\right)}{|N|^{2}} \leq \sum_{n_{i} \in N} \frac{\sum_{n_{j} \in N} r_{i j}\left(\mathscr{R}_{i}^{t *}\right)}{|N|^{2}} .
$$

Putting it in another way, Inequality (14) can be transformed into equation (15) as follows:

$$
\begin{aligned}
\mathscr{R}_{i}^{t *} & =\operatorname{argmax} \sum_{n_{i} \in N} \frac{\sum_{n_{j} \in N} r_{i j}\left(\mathscr{R}_{i}^{t}\right)}{|N|^{2}}, \\
\text { s.t. } \quad B_{i}^{t *} & =\left\{\left(\mathscr{R}_{i}^{t *}, \mathscr{P}_{i}^{t *}\right)\right\} .
\end{aligned}
$$

Therefore, we prove that $B_{i}^{t *}$ is the optimal solution in the feasible solution space of equation (1), where the objective function reaches the maximum value at the achieved consensus. Putting it in another way, the Nash equilibrium $B_{i}^{t *}=\left\{\left(\mathscr{R}_{i}^{t *}, \mathscr{P}_{i}^{t *}\right)\right\}$ is the optimal solution to the cooperative piggybacking problem in equation (1).

\section{Simulations}

6.1. Simulation Setup. In this section, we evaluate $\mathrm{CoB}$ with some real-world Wi-Fi communication data traces in the Isti/rural dataset. It is compared with the following broadcast schemes in terms of MDR, time delay, and communication overhead:
(1) Probabilistic Flooding $(P F)$. Each node forwards its received messages with a predefined probability (say $p=50 \%$ in our simulation) to improve MDR in the broadcast.

(2) Retransmission-Based Recovery $(R R)$. The node carries a request list in its message to inform others which one it has lost. The source node, i.e., the one that generated this message, will make the retransmission until it is received by all the nodes.

(3) Greedy Recovery (GR). Each node carries a request list in its packets. It also carries some messages to help other nodes recover their lost ones. The more the nodes request for recovering a message, the higher priority it will be carried for recovery first.

(4) Centralized Recovery (CR). Assume there exists a central node that can obtain the global information in the network. The central node develops the recovery strategy by using the global information and then disseminates it to the other nodes. These nodes recover the lost messages following the strategy developed by the central node.

Isti/rural contains a set of real-world communication data traces which records the link qualities within a Wi-Fi network. Each node in the Wi-Fi network is equipped with a CNet CNWLC-811 IEEE 802.11b PCMCIA wireless card and a standard driver in the ad hoc mode. Fragmentation, RTS/CTS, retransmissions, and dynamic rate switching are disabled and each message is only broadcast once. This helps to sample the link qualities quickly and accurately compared to the setup where retransmission is enabled after each message loss. For each data trace in the Isti/rural, it records the status of message delivery (which can be regarded as the link qualities) in the broadcast, where the distance between the sending node and the receiving node is set as a fixed value. If a message can be received successfully, the link quality is marked as " 1 "; otherwise, it is marked as " 0 ". Isti/rural is composed of a number of such data traces by varying the distance between the sending node and the receiving node with a difference of every 20 meters, starting from 40 to 300 meters. In our simulations, we set the gap between two adjacent spacer bars as 20 meters. For each wireless communication link in the broadcast, we allocate it with a unique data trace in the Isti/ rural, where the gap between the two spacer bars along the wireless communication link is equal to the distance between the sending node and the receiving node in the data trace. If the wireless communication link is reliable, i.e., the status of message delivery in the allocated data trace is marked as " 1 ," the simulator will deliver the message to the receiving node; otherwise, the simulator will discard the message. The TTL of messages is set as 100 milliseconds, and the nodes can carry at most two messages in each transmission.

6.2. Broadcast Reliability. As shown in equation (1), we calculate the MDR for each node in the broadcast, and define the average value of each message's MDR as the broadcast 


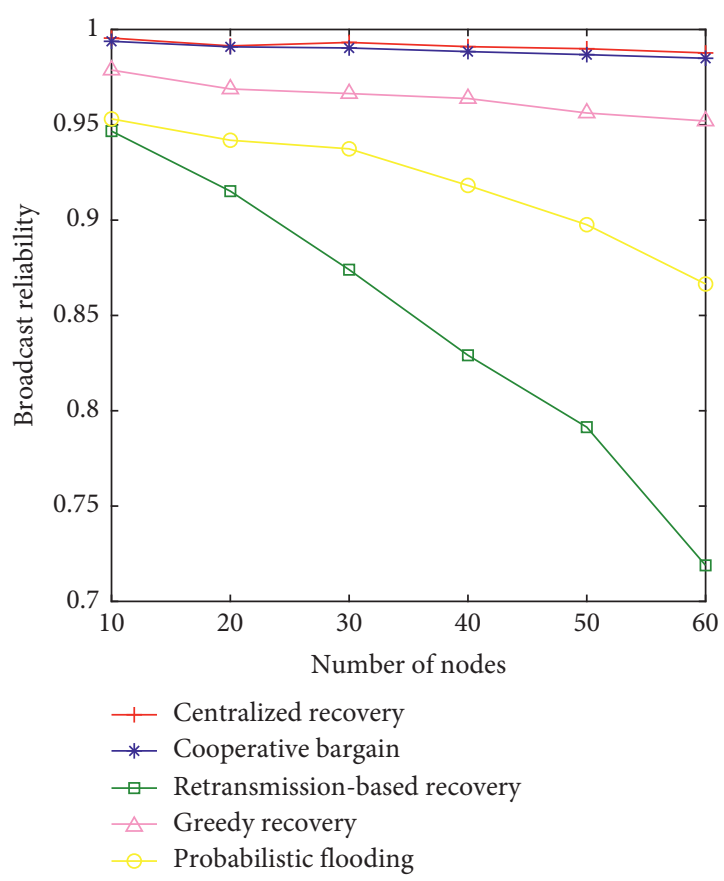

(a)

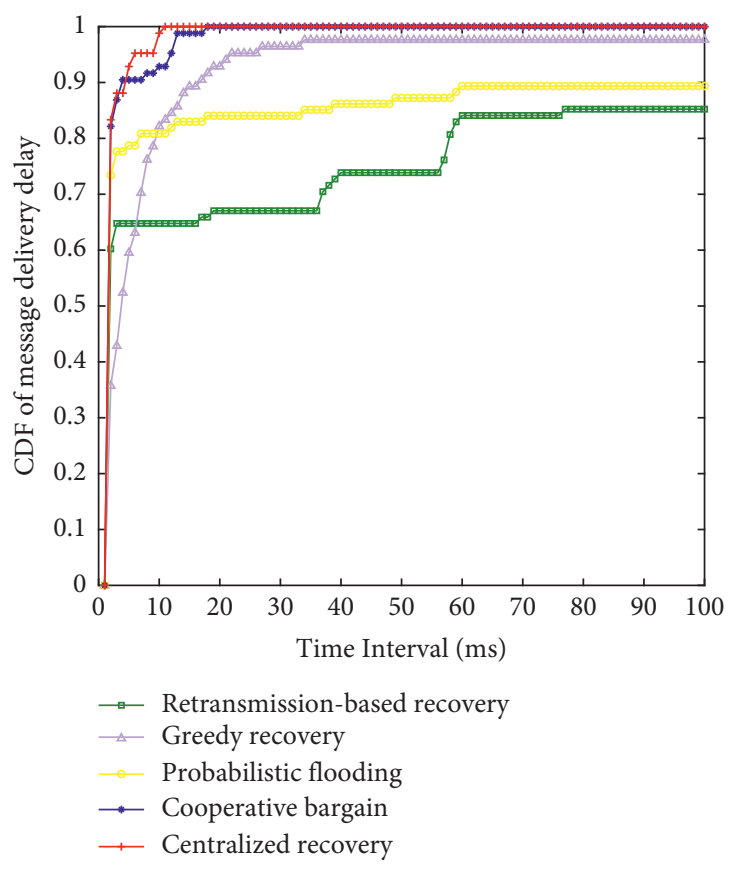

(c)

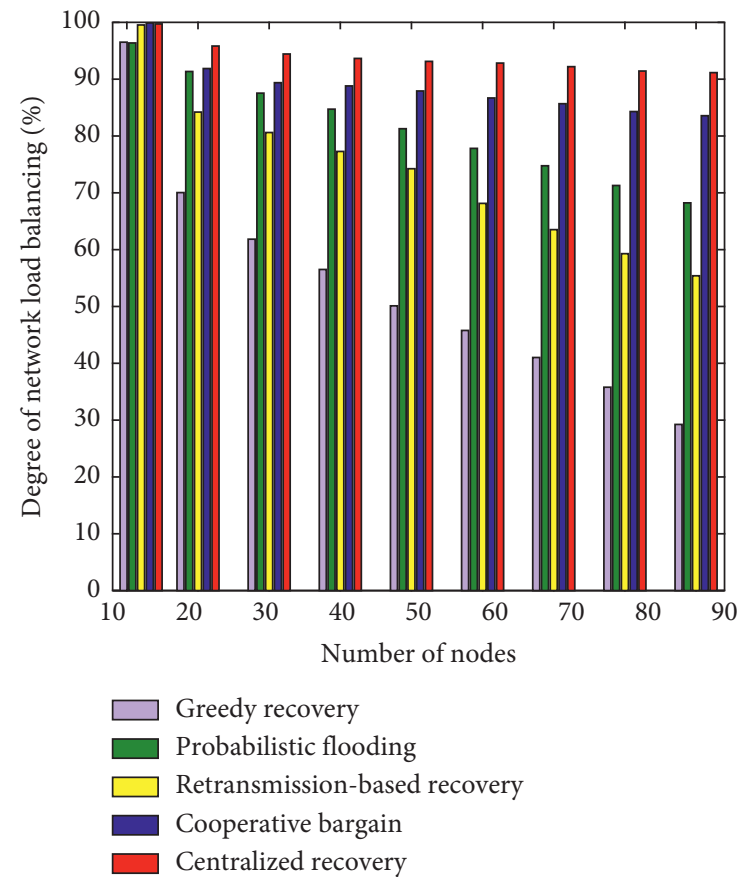

(b)

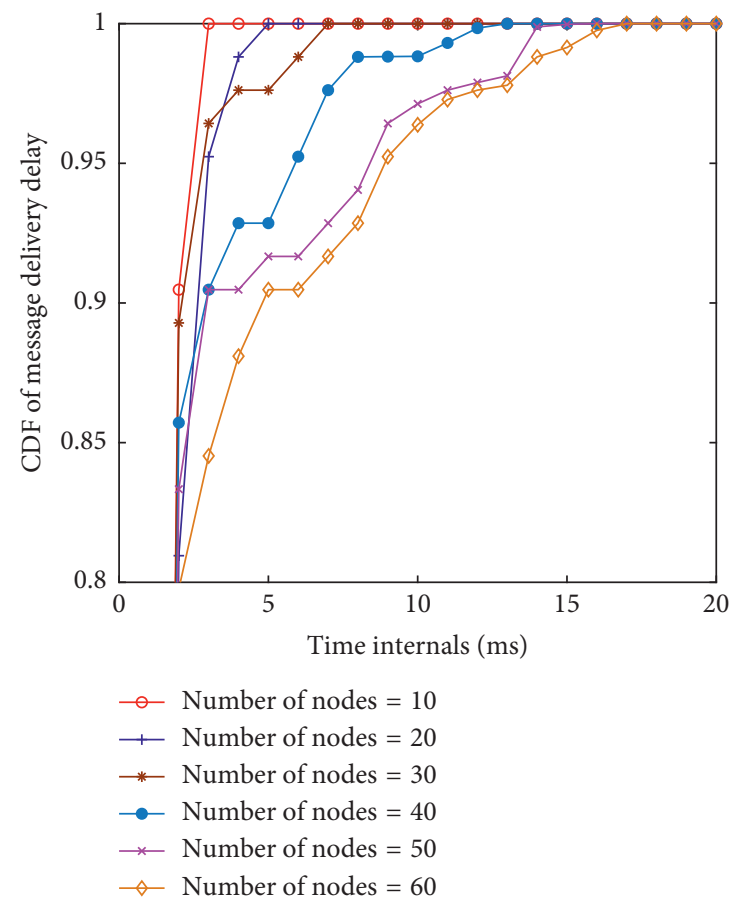

(d)

FIgURe 3: The performance evaluations on cooperative bargain. (a) Broadcast reliability. (b) Network load balancing. (c) Message delivery delay. (d) Recovery accuracy.

reliability. Figure 3(a) illustrates the MDR of different broadcast schemes when the number of nodes varies from 5 to 30. Since CP assumes all the nodes can acquire the global information, it has the highest MDR compared with other broadcast schemes. Note that the proposed CoB can achieve a comparable MDR to $\mathrm{CP}$, only using each node's local information for the recovery of lost messages. Moreover, when the number of nodes increases, the MDRs of CoB and $\mathrm{CP}$ are basically unchanged. It indicates that both schemes are stable and robust, which are little impacted by the number of nodes. GP follows behind $\mathrm{CoB}$ with an average MDR of $96 \%$, and it decreases slightly when the number of nodes increases. This mainly results from the greedy recovery of lost messages in GP, as the messages that are lost by 
TABLE 1: The average number of transmitted packets for each successfully received message.

\begin{tabular}{lcccccc}
\hline & \multicolumn{5}{c}{ Number of nodes } \\
& 4 & 6 & 8 & 10 & 12 & 14 \\
\hline CBP & 63 & 72 & 87 & 98 & 106 & 125 \\
CP & 87 & 111 & 125 & 169 & 234 & 271 \\
GP & 91 & 113 & 134 & 150 & 150 & 150 \\
SP & 181 & 285 & 337 & 377 & 436 & 475 \\
PF & 237 & 359 & 525 & 692 & 949 & 1157 \\
\hline
\end{tabular}

more nodes will be carried with higher priority, which can benefit more nodes in the recovery of lost messages. PF has a lower MDR (around 92\%) when the number of nodes is small, and it drops to $85 \%$ when the number of nodes increases to 30, as the redundant forwarding of messages may cause frequent data collisions and yield a high risk of overloading the communication channels in the broadcast. DR has the lowest MDR which drops significantly when the number of nodes increases from 5 to 30 .

6.3. Network Load Balancing. Let $\left|N_{i}\right|$ be the number of messages transmitted by vehicle $v_{i}$ in the broadcast, and $|\bar{N}|$ is the average number of transmitted messages at each node in the broadcast. Then, we define the degree of network load balancing (DNLB) as $\left(\sum_{i=1}^{|N|} P_{\Psi_{i}}\right)^{2} /|N| \cdot \sum_{i=1}^{|N|}\left(P_{\Psi_{i}}\right)^{2}$. If the nodes have transmitted the same number of messages in the broadcast, DNLB is maximized as 1 . If one node has transmitted all the messages, whereas the other nodes have transmitted no messages, DNLB is minimized as $1 / n$. As shown in Figure 3(b), CoB has a high DNLB which decreases slightly when the number of nodes increases. This results from the negotiation for eliminating the bias among the nodes where the task of message recovery is allocated regarding each node's local information. CP has a similar high DNLB as it assumes the central node can acquire the global information and thus make optimal recovery strategy with balanced network load. GP has the lowest DNLB as the nodes always tend to carry the same message for recovery, i.e., the one that is requested by most nodes, which will cause imbalanced network load in the nodes.

6.4. Delay of Message Delivery. We define the delay of message delivery (DMD) as the time interval between the instant when a message is generated and the instant when it is delivered or recovered at all the receiving nodes. Figure 3(c) shows the cumulative distribution function (CDF) of DMDs when the number of node is set as 30 . $\mathrm{CoB}$ and $\mathrm{CP}$ have the shortest $\mathrm{DMD}$ in the recovery of lost messages, which are always smaller than 15 milliseconds. GP fails to recover all lost messages, and the CDF climbs to 0.98 when the TTL expires. PF only recovers $85 \%$ lost messages at the end of TTL, as all the nodes forward their received packets which will cause severe data collisions in the broadcast. DR has the worst performance which only recovers $80 \%$ lost messages. This is because the success probability of message recovery highly depends on the qualities of wireless communication links, and retransmission helps little in the recovery of lost messages if the qualities of wireless communication links remain unstable.

Another factor that may impact the DMD is the network size, i.e., the number of nodes in the network. When the number of nodes is small, the nodes tend to have a low probability of data collisions when accessing the communication channel for broadcasting messages. In contrast, when the number of nodes increases, the nodes suffer from a high probability of data collisions when accessing the communication channel for broadcasting. This will also deteriorate the success probability in the recovery of lost messages and yield a long time delay in DMDs. As shown in Figure 3(d), the DMDs for recovering lost messages within a 5-node network are averagely shorter than $20 \mathrm{~ms}$, whereas the DMDs for recovering lost messages within a 30-node network rise to $75 \mathrm{~ms}$. The DMDs increase when the number of nodes increases, but $\mathrm{CoB}$ can still recover most messages before the TTLs expire.

6.5. Recovery Accuracy. We define the recovery accuracy as the average number of transmitted messages for each received message, that is, the ratio between the total number of transmitted messages and the number of successfully received messages. As shown in Table 1, fewer messages are transmitted in $\mathrm{CoB}$ compared to that of $\mathrm{CP}$. This is because the nodes in $\mathrm{CoB}$ work in a decentralized environment and they only use the local information to make cooperative piggybacking. In contrast, the central node in $\mathrm{CP}$ has to acquire the global information via exchanging messages with all the other nodes. After developing the optimal recovery strategy, the central nodes have to communicate with the other nodes again to disseminate the developed optimal recovery strategy, which will yield a large communication overhead in the broadcast. GP has transmitted more messages in the broadcast, and the number of transmitted packets increases when the network size becomes large. SP and $\mathrm{PF}$ have the worst performance in terms of network load, where 475 and 1157 packets are transmitted in each broadcast period, respectively, which may cause a high risk of data collisions in the broadcast.

\section{Conclusion}

This paper proposes a decentralized Cooperative Bargain scheme, namely $\mathrm{CoB}$, to improve the broadcast reliability when disseminating messages in the reversible lanes. It aims to drive the spacer bars make consistent movement for the autonomous separation of traffic flows. To achieve this target, each active spacer bar is allowed to carry some received messages to help others recover the lost ones. Besides, a cooperative bargain game is formulated to allocate the task of message recovery regarding each spacer bar's local information. Each active spacer bar keeps on negotiating with others and updating its recovery strategy until a consensus is achieved, which is proved to be an optimal solution to the recovery of lost messages. Our future work is to improve the convergence speed, that is, minimizing the process of 
negotiation in the recovery of lost messages, and extend the proposed scheme to other ad hoc networks.

\section{Data Availability}

The real-world Wi-Fi communication data trace (Isti/rural) used to support the findings of this study were supplied by RAWDAD (a community resource for archiving wireless data at Dartmouth, http://www.crawdad.org/isti/rural) under license and so cannot be made freely available. Requests for access to these data should be made to the web administrator via http://crawdad@crawdad.org. Isti/rural contains a group of dataset of transmission distance vs. packet loss measurement on a Wi-Fi network in rural areas. A series of measurements for relating transmission distance and packet loss on a Wi-Fi network in rural areas are conducted to propose a model that relates distance with packet loss probability. The data/time of the measurement was released on 2007-12-19, and the date/time of measurement started on 2005-03-25 and ended on 2006-04-23.

\section{Conflicts of Interest}

The authors declare that they have no conflicts of interest.

\section{Acknowledgments}

The authors would like to thank the National Natural Science Foundation of China (61803206), the Key R\&D Program of Jiangsu Province (BE2017008-2), the Nanjing Forestry University Youth Science and Technology Innovation Fund (CX2018004), and the Nanjing Forestry University Self-made Experimental Teaching Instrument Project (nlzzyq201827) for partly funding this project.

\section{References}

[1] A. M. Nagy and V. Simon, "Survey on traffic prediction in smart cities," Pervasive and Mobile Computing, vol. 50, pp. 148-163, 2018.

[2] Z. Vander Laan and K. F. Sadabadi, "Operational performance of a congested corridor with lanes dedicated to autonomous vehicle traffic," International Journal of Transportation Science and Technology, vol. 6, no. 1, pp. 42-52, 2017.

[3] V. A. Banks, N. A. Stanton, G. Burnett, and S. Hermawati, "Distributed cognition on the road: using EAST to explore future road transportation systems," Applied Ergonomics, vol. 68 , pp. 258-266, 2018.

[4] S. Zaidi, B. Hmidet, and S. Affes, "Distributed collaborative beamforming for real-world WSN applications," Ad Hoc Networks, vol. 184, pp. 316-329, 2017.

[5] C. Miao, H. Zhang, H. Zhou, P. Dong, and S. Shen, "Super node routing strategy in content-centric networking," Transactions of Tianjin University, vol. 21, no. 2, pp. 122-128, 2015.

[6] Y. Wu, L. Ming, and X. Ao, "QoS-aware directional flooding based on redundant route for mobile ad hoc network," Journal of Internet Technology, vol. 18, no. 3, pp. 677-684, 2017.

[7] F. J. Martin-Vega, B. Soret, M. C. Aguayo-Torres, I. Z. Kovacs, and G. Gomez, "Geolocation-based access for vehicular communications: analysis and optimization via stochastic geometry," IEEE Transactions on Vehicular Technology, vol. 67, no. 4, pp. 3069-3084, 2018.

[8] H. Ko and S. Pack, "Phase-aware directional energy transmission algorithm in multiple directional RF energy source environments," IEEE Transactions on Vehicular Technology, vol. 68, no. 1, pp. 359-367, 2019.

[9] M. Min, L. Xiao, Y. Chen, P. Cheng, D. Wu, and W. Zhuang, "Learning-based computation offloading for IoT devices with energy harvesting," IEEE Transactions on Vehicular Technology, vol. 68, no. 2, pp. 1930-1941, 2019.

[10] H. M. A. Aziz, F. Zhu, and S. V. Ukkusuri, "Learning-based traffic signal control algorithms with neighborhood information sharing: an application for sustainable mobility," Journal of Intelligent Transportation Systems, vol. 22, no. 1, pp. 40-52, 2018.

[11] H. Jin and P. Papadimitratos, "DoS resilient cooperative beacon verification for vehicular communication systems," Ad Hoc Networks, vol. 90, Article ID 101775, 2018.

[12] S. Kim, "Timed bargaining-based opportunistic routing model for dynamic vehicular ad hoc network," EURASIP Journal on Wireless Communications and Networking, vol. 14, 2016.

[13] N. W. Prasetya, T.-L. Sheu, A. Basuki, and M. A. Muslim, “A pre-emptive traffic model with cluster-based TDMA for vehicular networks," in Proceedings of the 2016 24th International Conference on Software, Telecommunications and Computer Networks (SoftCOM), pp. 1-5, Split, Croatia, September 2016.

[14] L. Mottola, G. P. Picco, M. Ceriotti, Ş. Gună, and A. L. Murphy, "Not all wireless sensor networks are created equal: a comparative study on tunnels," ACM Transactions on Sensor Networks, vol. 7, no. 2, 2010.

[15] F. Saffre, R. Tateson, and R. Ghanea-Hercock, "Reliable sensor networks using decentralised channel selection," Computer Networks, vol. 46, no. 5, pp. 651-663, 2004.

[16] B. Yuan, A. Jie, and Z. Huibing, "Location aided probabilistic broadcast algorithm for mobile Ad-hoc network routing," The Journal of China Universities of Posts and Telecommunications, vol. 24, no. 2, pp. 66-71, 2017.

[17] S. Dash, B. J. R. Sahu, N. Saxena, and A. Roy, "Flooding control in named data networking," IETE Technical Review, vol. 35, no. 3, pp. 266-274, 2018.

[18] S.-G. Byeon, H.-Y. Seo, and J.-D. Kim, "Contribution-levelbased opportunistic flooding for wireless multihop networks," Journal of KIISE, vol. 42, no. 6, pp. 791-800, 2015.

[19] A. Bazzi and A. Zanella, "Position based routing in crowd sensing vehicular networks," Ad Hoc Networks, vol. 36, pp. 409-424, 2016.

[20] G. Xiao, H. Zhang, Z. Huang, and Y. Chen, "Decentralized cooperative piggybacking for reliable broadcast in the VANET," in Proceedings of the 2016 IEEE 83rd Vehicular Technology Conference, Nanjing, China, May 2016.

[21] M. Xu, Q. Yang, and K. S. Kwak, "Distributed topology control with lifetime extension based on non-cooperative game for wireless sensor networks," IEEE Sensors Journal, vol. 16, no. 9, pp. 3332-3342, 2016.

[22] S. Kaul, R. Yates, and M. Gruteser, "On piggybacking in vehicular networks," in Proceedings of the IEEE Global Telecommunications Conference (GLOBECOM 2011), vol. 1, pp. 1-5, Houston, TX, USA, December 2011.

[23] G. Song, J. Rochas, F. Huet, F. Lea, F. Magoules, and Magoules, "K nearest neighbour joins for big data on MapReduce: a theoretical and experimental analysis," IEEE 
Transactions on Knowledge and Data Engineering, vol. 28, no. 9, pp. 2376-2392, 2016.

[24] F. Librino, M. E. Renda, and P. Santi, "Multihop beaconing forwarding strategies in congested IEEE 802.11p vehicular networks," IEEE Transactions on Vehicular Technology, vol. 65, no. 9, pp. 7515-7528, 2016.

[25] K. Alodadi, A. H. Al-Bayatti, and N. Alalwan, "Cooperative volunteer protocol to detect non-line of sight nodes in vehicular ad hoc networks," Vehicular Communications, vol. 9, pp. 72-82, 2017.

[26] G. Xiao, N. Sun, L. Lv, Y. Ma, and Y. Chen, "An HEED-based study of cell-clustered algorithm in wireless sensor network for energy efficiency," Wireless Personal Communications, vol. 81, no. 1, pp. 373-386, 2015.

[27] F. Li, C. Tian, T. Li, and Y. Wang, "Energy efficient social routing framework for mobile social sensing networks," Tsinghua Science and Technology, vol. 21, no. 4, pp. 363-373, 2016.

[28] G. Xiao, H. Zhang, Z. Huang, and Y. Chen, "Decentralised data piggybacking and link scheduling for reliable broadcast in VANETs," in Proceedings of the 15th ACM International Symposium on Mobility Management and Wireless Access, pp. 33-40, Miami, FL, USA, November, 2017.

[29] K. S. Bilstrup, E. Uhlemann, and E. G. Strom, "Scalability issues of the MAC methods STDMA and CSMA of IEEE $802.11 \mathrm{p}$ when used in VANETs," in Proceedings of the IEEE International Conference on Communications Workshops (ICC), pp. 1-5, Cape Town, South Africa, May 2010.

[30] D. Guerrero, A. A. Carsteanu, and J. B. Clempner, "Solving Stackelberg security Markov games employing the bargaining Nash approach: convergence analysis," Computers \& Security, vol. 74, pp. 240-257, 2018. 


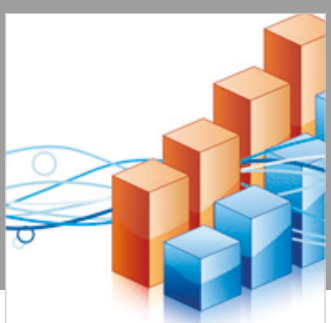

Advances in

Operations Research

\section{-n-m}
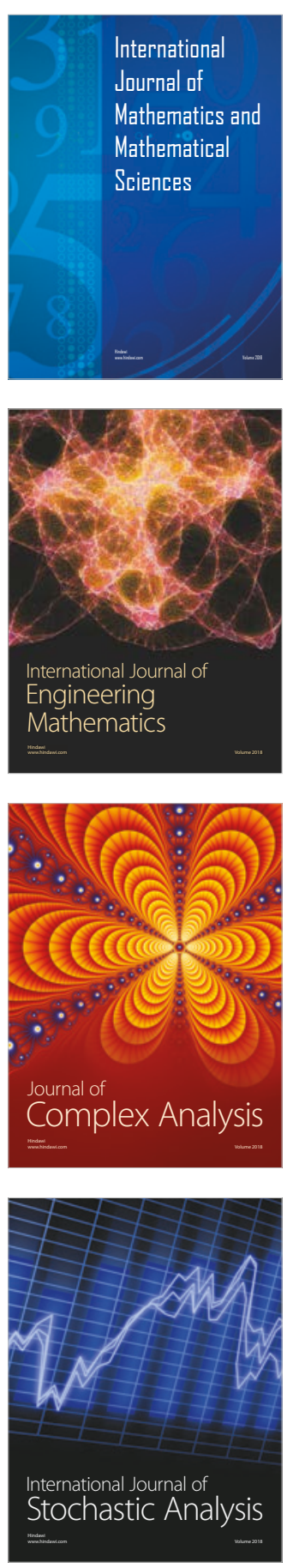
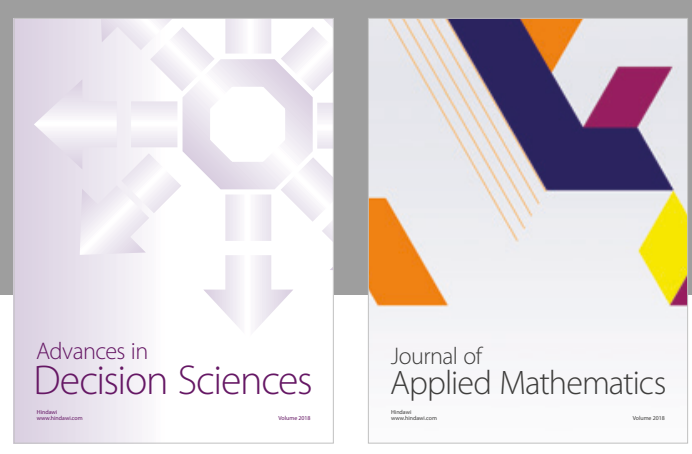

Journal of

Applied Mathematics
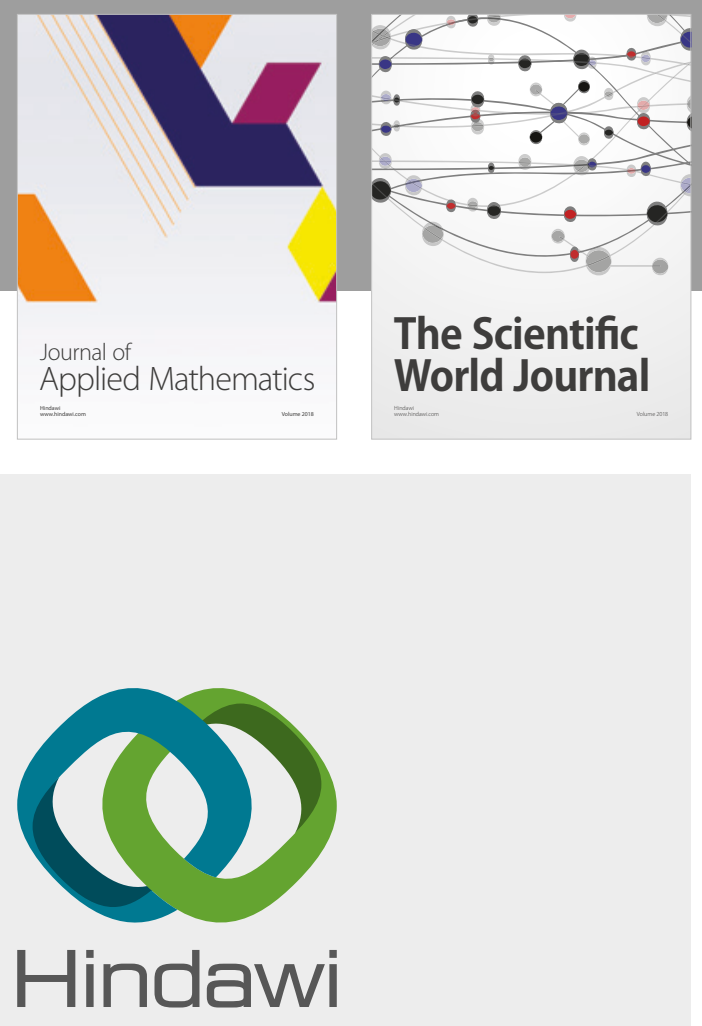

Submit your manuscripts at

www.hindawi.com

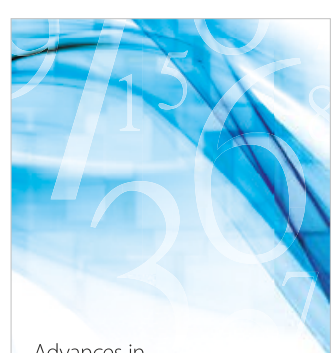

Advances in
Numerical Analysis
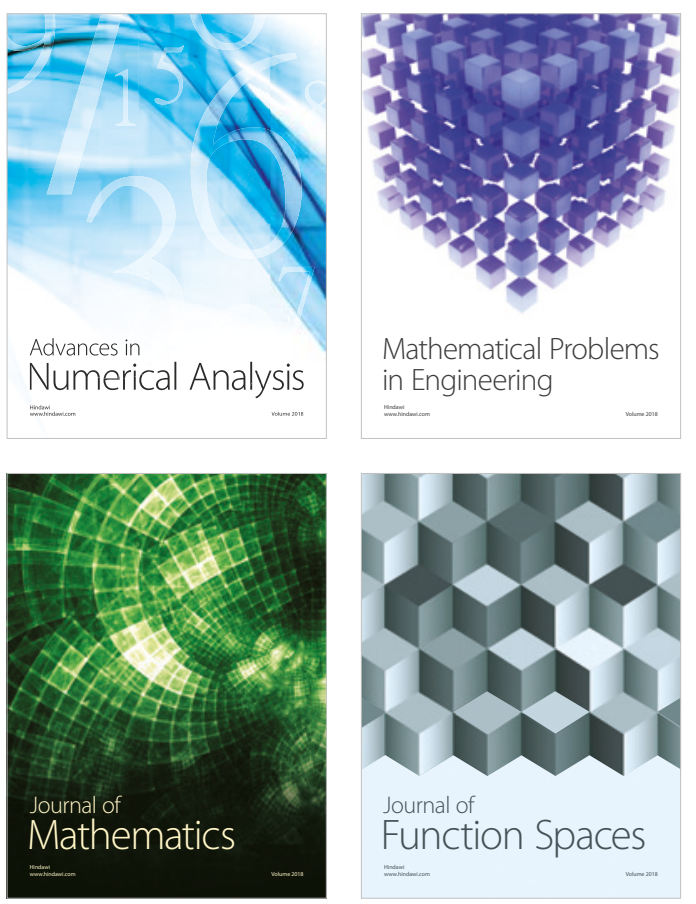

Mathematical Problems in Engineering

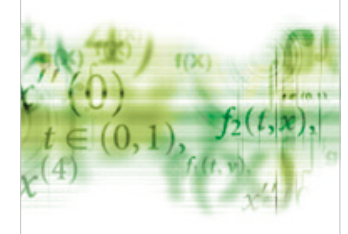

International Journal of

Differential Equations

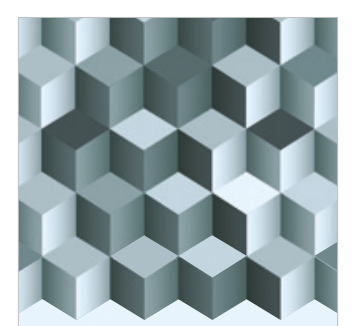

Journal of

Function Spaces
The Scientific

World Journal

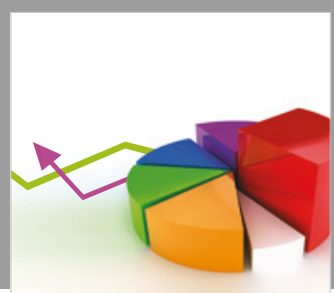

Journal of

Probability and Statistics
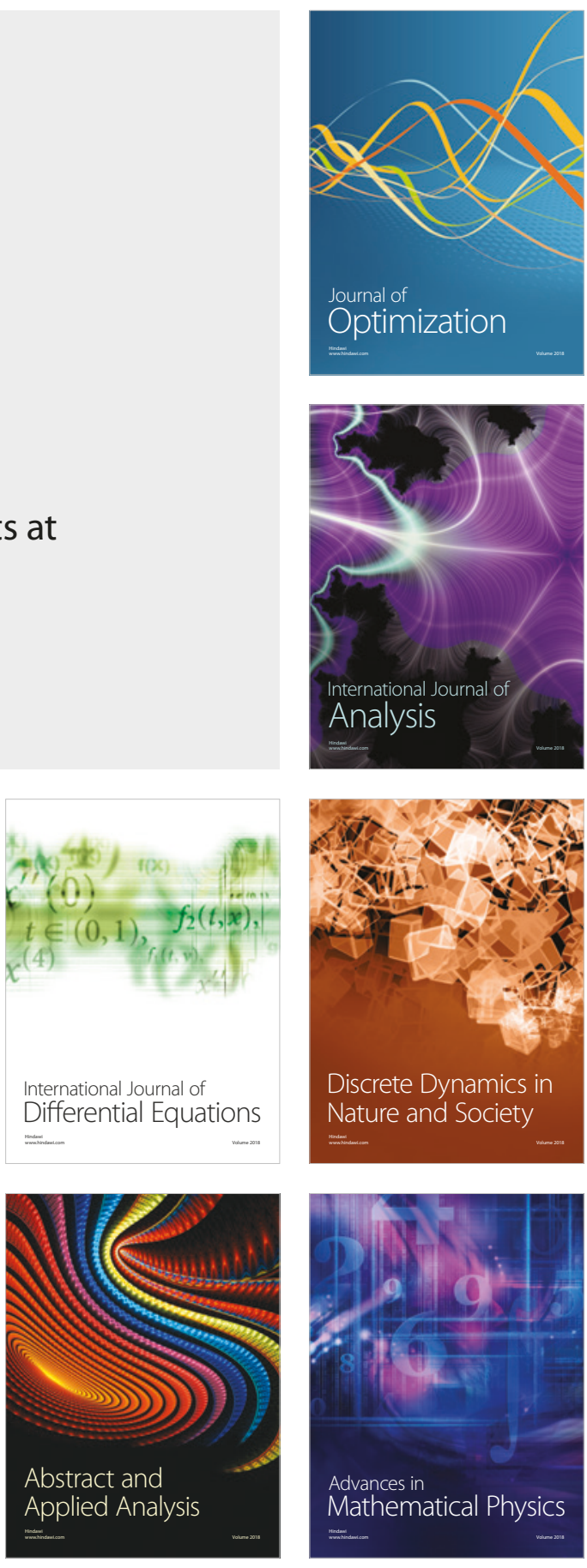